\title{
Predicción de mortalidad perioperatoria en pacientes sometidos a cirugía vascular no cardíaca
}

\section{Objetivo}

Desarrollar un índice simple que contemple los factores de riesgo clínicamente significativos y la medicación crónica, para la predicción de la mortalidad perioperatoria en los pacientes sometidos a cirugía vascular mayor.

\section{Diseño}

Desarrollo $(n=1537)$ y validación $(n=773)$ de una regla de predicción* de mortalidad perioperatoria en pacientes sometidos a cirugía vascular no cardíaca electiva o de urgencia.

\section{Lugar}

Erasmus Medical Center, Rotterdam.

\section{Pacientes}

2310 pacientes (75\% hombres, edad media 68 años) sometidos a cirugía vascular no cardiaca, electiva o de urgencia entre 1991 y 2000.

\section{Métodos}

Factores de riesgo seleccionados: edad mayor a 70, angor previo o actual, historia de infarto de miocardio (IM) insuficiencia cardíaca (IC), accidente cerebrovascular (ACV) o accidente isquémico transitorio (AIT), diabetes, hipertensión, enfermedad pulmonar crónica disfunción renal y prescripción de fármacos crónicos.

La medida de resultado fue la mortalidad de cualquier causa, ocurrida antes del alta o dentro de los 30 días después de la cirugía. El $67 \%$ de los pacientes conformó la cohorte de desarrollo de la regla partiendo del índice de Lee'. Se elaboró un índice simplificado mediante regresión logística, validado en el resto de la cohorte.

\section{Resultados}

La mortalidad perioperatoria fue similar en las cohortes de desarrollo $(6,7 \%)$ y validación $(6,5 \%)$. La probabilidad de muerte perioperatoria se incrementó progresivamente en los pacientes sometidos a cirugía de ruptura aguda de aneurisma de aorta abdominal, toracoabdominal y abdominal, comparados con la endarterectomía carotídea; y se redujo con el uso de b bloqueantes y estatinas (ver cuadro 1). Mediante el puntaje total de los predictores puede estimarse la probabilidad de muerte (figura 1).
Cuadro 1: predictores independientes de mortalidad perioperatoria en pacientes sometidos a cirugía cardiovascular.

\begin{tabular}{|c|c|c|c|c|}
\hline & \multirow[t]{2}{*}{ Predictor independiente } & \multirow[b]{2}{*}{ Puntaje } & \multicolumn{2}{|c|}{ OR en cohorte } \\
\hline & & & Desarrollo & Validacion \\
\hline \multirow{4}{*}{$\begin{array}{l}\text { Tipo de } \\
\text { cirugía }\end{array}$} & Ruptura Abdominal & 43 & $\mid 171,3(19,3-262,8)$ & $40(8,7-183,3)$ \\
\hline & \begin{tabular}{|l|l|} 
aórtica & Torácica y/o torácoabdominal \\
\end{tabular} & 26 & $13,1(3,6-47,7)$ & $11(2,6-46,5)$ \\
\hline & Bypass infrainguinal & 15 & $4,4(1,1-16,8)$ & $5,8(1,3-24,8)$ \\
\hline & Endarterectomía carotídea & 0 & 1(referencia) & 1(referencia) \\
\hline \multirow{6}{*}{$\begin{array}{l}\text { Morbilidad } \\
\text { cardio } \\
\text { vascular }\end{array}$} & Infarto de miocardio & 13 & $3,5(2,1-6)$ & $2,3(1,1-4,7)$ \\
\hline & Insuficiencia cardíca & 14 & $4(1,9-7,7)$ & $3,5(1,5-8,6)$ \\
\hline & Accidente cerebrovascular & 10 & $2,7(1,5-4,7)$ & $6,5(2,8-15)$ \\
\hline & Hipertensión arterial & 7 & $2(1,2-3,3)$ & $1,4(0,7-2,7)$ \\
\hline & Enfermedad renal & 16 & $5,2(2,6-10,2)$ & $6,7(2,9-15,1)$ \\
\hline & EPOC & 7 & $2(1,1-3,4)$ & $2,4(1,1-5,1)$ \\
\hline \multirow{2}{*}{$\begin{array}{l}\text { Fármacos } \\
\text { crónicos }\end{array}$} & $\beta$ bloqueantes & -15 & $0,2(0,1-0,5)$ & $0,3(0,1-0,7)$ \\
\hline & estatinas & -10 & $0,4(0,2-0,8)$ & $0,2(0-0,09)$ \\
\hline
\end{tabular}

Figura 1: probabilidad de muerte perioperatoria según el puntaje resultante de los predictores clínicos.

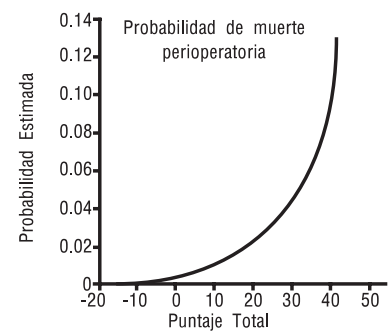

\section{Conclusiones}

La combinación de predictores clínicos, el tipo de cirugía y la terapia con $b$ bloqueantes y/o estatinas permiten estimar la probabilidad de mortalidad perioperatoria en pacientes sometidos a cirugía vascular electiva o de urgencia.

Fuente de financiamiento: no referida.

\section{Comentario}

El trabajo desarrolla y valida un índice de riesgo de mortalidad perioperatoria de cualquier causa a partir de un índice de riesgo cardíaco en cirugía no cardiaca, el índice de Lee, en una cohorte de pacientes sometidos a procedimientos vasculares (no cardíacos). El índice de Lee fue desarrollado sobre pacientes mayores de 50 años sometidos a procedimientos mayores no cardíacos en forma electiva, y la medida de resultado consistió en complicaciones cardíacas mayores (definidas como IM, edema agudo de pulmón, fibrilación ventricular/ paro cardíaco primario y bloqueo AV completo). Se concibe este nuevo índice para estimar la mortalidad global, agregando otros datos médicos como EPOC (predictor de complicaciones respiratorias ${ }^{2,3}$ ) pero tanto el desarrollo como la validación, se realizan sobre una población de pacientes vasculares. El nivel de optimización logrado en el modelo final depende en gran parte del tipo de procedimiento, definido como de alto riesgo cuando se trata de una cirugía de aneurisma de aorta (OR 71). Por otro lado, el antecedente de AIT aparece como protector, lo cual es difí- cil de explicar. Podría significar que estos pacientes tienen una mayor intensidad de cuidado médico, o tal vez sea un factor confundidor. El uso de b bloqueantes y estatinas aparece como protector, algo que Lee et al no pudieron encontrar en una población más gene-ral, posiblemente por falta de poder'. La suspensión de la aspirina no aumentó el riesgo de mortalidad.

\section{Conclusiones del comentador}

El trabajo desarrolla un índice de riesgo de mortalidad general en una cohorte de pacientes sometidos a procedimientos vasculares, colocando una lente de aumento sobre el grupo de mayor mortalidad en el trabajo de Lee et al. La terapia con b bloqueantes y estatinas constituyen factores protectores, lo cual es esperable en pacientes panvasculares.

\section{Daniel Casas y María Maragoto [ Servicio de Anestesiología, Hospital Italiano de Buenos Aires. ]}

Casas DM, Maragoto M. Predicción de la mortalidad perioperatoria en cirugía vascular con un índice simple. Evid actual pract ambul 2005;107. Kertai MD, Boersma E, Klein J, et al. Optimizing the prediction of perioperative mortality in vascular surgery by using a customized probability model. Arch Intern Med. 2005;165:898-904. PMID: 15851641. Erratum in: Arch Intern Med. 2005;165:1540.

\section{Referencias:}

1. Lee TH, Marcantonio ER, Mangione CM, Thomas EJ, Polanczyk CA, Cook EF, Sugarbaker DJ, Donaldson MC, Poss R, Ho KK, Ludwig LE, Pedan A, Goldman L. Derivation and prospective validation of a simple index for prediction of cardiac risk of major noncardiac surgery. Circulation. 1999;100:1043-9.

2. Arozullah AM, Khuri SF, Henderson WG, Daley J, et al. Development and validation of a multifactorial risk index for predicting postoperative pneumonia after major noncardiac surgery. Ann Intern Med. 2001;135:847-57.

3. Arozullah AM, Daley J, Henderson WG, Khuri SF. Multifactorial risk index for predicting postoperative respiratory failure in men after major noncardiac surgery. Ann Surg. 2000;232:242-53. 